\title{
Fabrication and Packaging of a Fully Implantable Biosensor Array
}

\author{
Camilla Baj-Rossi**, Enver G. Kilinc ${ }^{\dagger}$, Sara S. Ghoreishizadeh*, Daniele Casarino ${ }^{\ddagger}$, Tanja Rezzonico Jost ${ }^{\S}$, \\ Catherine Dehollain ${ }^{\dagger}$, Fabio Grassi ${ }^{\S}$, Laura Pastorino ${ }^{\ddagger}$, Giovanni De Micheli* and Sandro Carrara* \\ ${ }^{*}$ Laboratory of Integrated Systems Swiss Federal Institute of Technology (EPFL), Lausanne, Switzerland \\ ${ }^{\dagger}$ RFIC Group, Swiss Federal Institute of Technology (EPFL), Lausanne, Switzerland \\ $\ddagger$ Universitá degli Studi di Genova, Italy \\ $\S$ Institute for Research in Biomedicine - IRB, Bellinzona, Switzerland \\ *Email: camilla.baj-rossi@epfl.ch
}

\begin{abstract}
In this work, we showed the realization of a fullyimplantable device that integrates a microfabricated sensing platform, a coil for power and data transmission and integrated circuits. We described a device intended to test the biocompatibility of the materials used for the microfabrication. Therefore, electronics measurements for data communication and remote powering will be reported in another article [1]. To ensure biocompatibility an epoxy enhanced polyurethane membrane was used to cover the device. We proved through an in-vitro characterization that the membrane was capable to retain enzyme activity up to 35 days. After 30 days of implant in mice, in-vivo experiments proved that the membrane promotes the integration of the sensor with the surrounding tissue, as demonstrated by the low inflammation level at the implant site.
\end{abstract}

\section{INTRODUCTION}

Electrochemical sensors for real-time monitoring of glucose and lactate are already in the market [2], moreover for glucose, first prototypes of fully implantable sensors have been validated up to 8 months in mice [3] and up to one year in pigs [4]. The development of an implantable device for monitoring exogenous substances, such as drugs and other important metabolites, would represent a big step towards personalized medicine. A device capable to monitor multiple substances should contain an array of sensors with enough sensitivity to monitor drugs and metabolites within the therapeutic range. Moreover, an implantable device should be autonomous with respect to the power supply of the data transmission block and of the sensor block including its electronic front-end. The biocompatibility and the sensor stability in time are other fundamental issues for implantable devices: the sensor should be well tolerated by the host and cause a limited foreign body reaction, and at the same time the contact with biological fluids should not significantly affect sensor performances [5]. The sensor performance may be also improved using nanostructured materials. Excellent performances have been obtained by using carbon nanotubes [6], metallic [7] or semiconducting [8] quantum dots or other nano-materials [9]. However, since carbon nanotubes may be potentially toxic, the biocompatibility of devices incorporating nanomaterials needs to be investigated.

The present work focuses on the design and fabrication of a prototype for a fully-implantable device that consists of: 1) a passive sensing platform, 2) integrated circuits to perform electrochemical measurements and 3) a coil for autonomy power transmission. We show the assembly of these components and the use of an innovative biocompatible packaging based on an epoxy enhanced polyurethane membrane. To test the properties of the membrane, we performed in-vitro characterization on screen-printed electrodes (SPEs) and in-vivo biocompatibility tests, by implanting the devices in mice for 30 days.

\section{Materials And Methods}

\section{A. Passive Chip Microfabrication}

Microfabrication was realized at the EPFL Centre of Micronano Technology (CMI). Silicon wafers with $500 \mathrm{~nm}$ of native oxide were chosen as substrate. Chip metalization was realized by evaporation of $10 \mathrm{~nm}$ of $\mathrm{Ti}$, followed by $100 \mathrm{~nm}$ of Pt. Metal passivation was made via atomic layer deposition of $\mathrm{Al}_{2} \mathrm{O}_{3}$, followed by dry etching with Argon Ion Milling. Details on the microfabrication can be found in [10].

\section{B. Sensors Functionalization and Membrane Deposition}

We performed electrochemical measurements with both carbon paste screen-printed electrodes (SPEs, model DRP110, DropSens) and the microfabricated passive platform. The working electrode of SPEs was nanostructured with $30 \mu \mathrm{g}$ multi-walled carbon nanotubes (MWCNTs, purchased by Dropsens), as reported in [11]. For the microfabricated platform, the working electrodes were functionalized by electrodeposition of a chitosan/MWCNT dispersion $8 \mathrm{mg} / \mathrm{ml}, \mathrm{pH}$ 5 , by applying a fixed potential of $1.5 \mathrm{~V}$ for $2400 \mathrm{~s}$ [10]. A solution of glucose oxidase enzyme (from Roche) in phosphate buffered saline (PBS) $1 \mathrm{X} \mathrm{pH} \mathrm{5.8,} \mathrm{was} \mathrm{drop} \mathrm{cast} \mathrm{on} \mathrm{the} \mathrm{working}$ electrodes and stored overnight at $4{ }^{\circ} \mathrm{C}$.

An epoxy polyurethane membrane was employed as protective membrane for the sensor [3]. A homogeneous solution was obtained by mixing $12.5 \mathrm{mg}$ of an epoxy adhesive (EP42HT-2Med system), purchased by Master Bond as a certified biocompatible two-components adhesive, $12 \mathrm{mg}$ of Polyurethane (PU, Sigma Aldrich), $1 \mathrm{mg}$ of the surfactant agent Polyethylene glycol ether (Brij ${ }^{\circledR}$ 30, Sigma Aldrich), for $1 \mathrm{ml}$ of Tetrahydrofuran (THF, Sigma Aldrich) used as solvent. The deposition method is known as dip coating and it consists 
of a quick immersion of the sensor in the membrane solution. Subsequent depositions were applied at $2 \mathrm{~h}$ intervals and then the sensors were stored overnight at room temperature. A fast curing at high temperature $\left(80^{\circ} \mathrm{C}\right)$ is needed to ensure the biocompatibility of the resin. After this process the sensors were again kept overnight at room temperature, and then stored in PBS one day for membrane swelling. When not in use, the sensors were stored in PBS at room temperature.

\section{Electrochemical Measurements}

Electrochemical measurements were performed using an Autolab electrochemical workstation (Metrohm). Electrodes were tested for glucose sensitivity with chronoamperometry at $+650 \mathrm{mV}$. The sensors were first dipped in a 1X PBS solution ( $\mathrm{pH} 7.4$ ), under stirring conditions, then conditioned for $1 \mathrm{~h}$ at $+650 \mathrm{mV}$ and then tested against repeated injections of glucose $100 \mu \mathrm{M}$. Sensitivity per unit area and limit of detection (LOD) were computed from the straight line obtained by plotting the current vs. glucose concentration, according to [11], [12].

\section{Integrated Device Assembly and Packaging}

The epoxy adhesive (EP42HT-2Med system) was used to assembly the electronic component in the integrated device. The interconnections between the pads of the passive chip and electronic components were realized with $\mathrm{Al}$ wire bonding and were protected with a glob top protection of $0.3 \mathrm{~mm}$. All the edges of the device were rounded with a milling machine. Two subsequent $5 \mu \mathrm{m}$ layers of Parylene $\mathrm{C}$ were deposited by chemical vapor deposition using a Comelec C-30-S Parylene Deposition System. Parylene C was used to cover the whole device but not the electrode array that needs to be in contact with fluids.

\section{E. In-vivo biocompatibility}

Microchips were cleaned and disinfected with ethanol $70 \%$ and placed in sterile PBS (Gibco) for $24 \mathrm{~h}$ to stabilize the membrane. An Air Pouch (AP) was created by subcutaneous injection of sterile air in the back of male C57BL/6 mice at day $1(5 \mathrm{~mL})$ and day $3(3 \mathrm{~mL})$ : this procedure creates a cavity of $1.5 \mathrm{~cm}$ diameter and $0.5 \mathrm{~cm}$ height. At day 6 , mice were anesthetized with isoflurane $4 \%$, shaved and locally sterilized with Betadine solution; the sterile microchips were implanted and the cavity sutured with Vicryl 6.0 (Provet AG). As a control of local inflammation, bacterial lipopolysaccharide (LPS) $(50 \mu \mathrm{g} / \mathrm{mouse})$ (LabForce AG) was injected daily into the cavity for the last 2 weeks of the 30 days of the experiment. As negative control APs were generated in the absence of any surgical procedure. As further control commercial chips (DATA MARS) were injected through sterile needle. After 30 days, the microchips were removed. The cavity was rinsed with $0.5 \mathrm{~mL}$ of PBS (Gibco) and the liquid collected and centrifuged at $7000 \mathrm{rpm}$ for $10 \mathrm{~min}$ at $4^{\circ} \mathrm{C}$. The concentration of ATP was determined in the supernatant with ATP determination kit (Invitrogen). For polymorphonuclear neutrophils detection, the pellet was resuspended in $0.2 \mathrm{~mL}$ RPMI $10 \%$ fetal bovine serum (Gibco) and analyzed at flow cytometer (FACS Canto, Becton Dickinson) with antibodies specific for

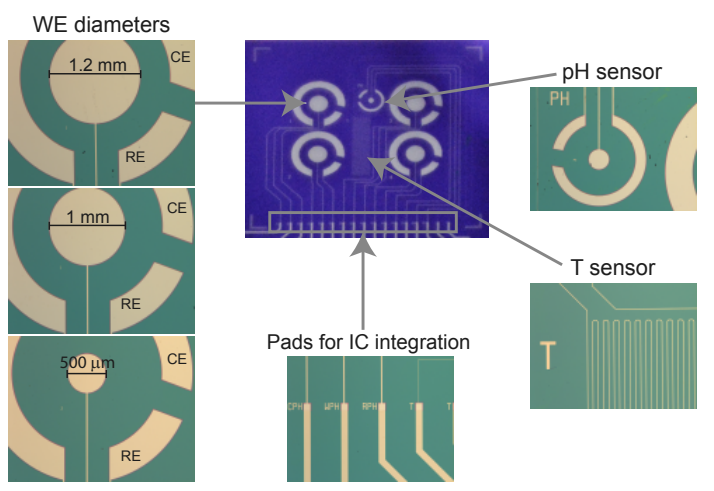

Fig. 1. Photographs of the microfabricated platform (center), with the three geometries for the working electrode (WE), the pads for integration with ICs and the $\mathrm{pH}$ sensor and the temperature sensor.

CD11b and Gr1, labelled with allophycocyanin (APC) and fluorescein isothiocyanate (FITC) (BioLegend), respectively.

\section{RESUltS AND Discussion}

\section{A. Implantable sensor design and fabrication}

1) Design and fabrication of the passive chip: The microfabrication of the passive chip was realized with a twomasks process flow. Details on the process flow can be found in [10]. The design of the passive chip was done under the constraints given by the compatibility with the other components. The platform measures $12 \times 11 \mathrm{~mm}$ in order to fit the size of the coil $(12 \times 12 \mathrm{~mm})$ and the wire bonding. Fig. 1 shows the photograph of the passive chip with the main structures. The platform hosts an array of four independent cells in the three-electrode configuration (working electrode (WE), counter electrode (CE) and a reference electrode (RE)). Working electrodes were realized in three different geometries (diameter of $500 \mu \mathrm{m}, 1 \mathrm{~mm}$ and $1.2 \mathrm{~mm}$ ). A pH sensor and a temperature sensor were fabricated in $\mathrm{Pt}$. The $\mathrm{pH}$ sensor has a WE with a $300 \mu \mathrm{m}$ diameter and it is based on an anodic iridium oxide film as reported in [10]. The resistive thermal device (RTD) consists of a Pt coil (4 $\mathrm{nm}$ width and $93 \mathrm{~nm}$ length) with an average resistivity of $35 \mathrm{k} \Omega$ at $20^{\circ} \mathrm{C}$, compatible with the power constraints. The main advantage behind the design of an array of independent sensors is the possibility to perform multiplexed measurements of drugs or metabolites. The design of a sensing platform for electrochemical measurement, combined with the presence of a $\mathrm{pH}$ and a temperature sensor, is a strategy to optimize the sensing performance in different physiological conditions, since changes in $\mathrm{pH}$ and temperature can affect the sensor specificity, e.g. in drug detection [13].

2) Measurement IC: A configurable integrated circuit has been designed and fabricated in $180 \mathrm{~nm}$ technology to control and readout the biosensor array. Readout circuit for the $\mathrm{pH}$ sensor and Pt-RTD are also integrated [14]. The readout IC will be powered by the power management IC.

3) Power Management: The power is transferred to the battery-less implanted system via an optimized inductively coupled power link over $30 \mathrm{~mm}$ distance. Fig. 2 (a) shows 


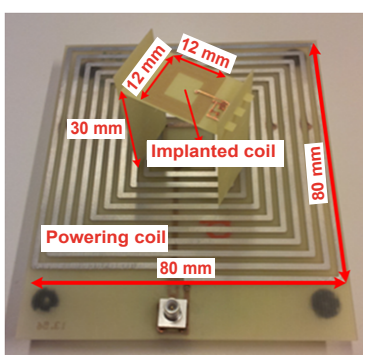

(a) Optimized power link.

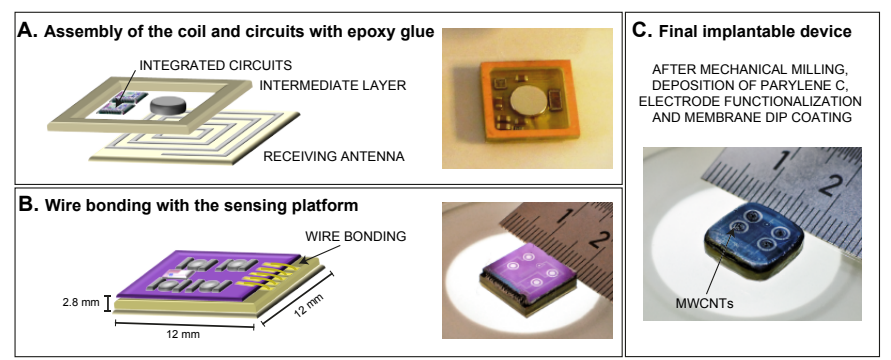

(b) Scheme of the integrated device assembly.

Fig. 2. Components of the implantable device.

the optimized inductively coupled power link. The sizes of the powering and implanted coils are $80 \times 80 \mathrm{~mm}$ and $12 \times 12$ $\mathrm{mm}$ respectively. The supply voltage of the overall system is generated by the power management circuits located above the implanted coil as shown in Fig. 2 (a). The power management circuits include the full-wave passive rectifier and the highspeed voltage regulator to supply a clean and stable $1.8 \mathrm{~V}$ supply voltage [15]. The biosensor system is intended to be implanted inside a freely moving animal. Therefore, an Intelligent Remote Powering (IRPower) system is designed to monitor the animal in the cage [16], and to adapt the transferred power level according to the position of the animal. Fig. 2 (b) shows the components of the implantable device, and the assembly steps: the integrated circuits and the coil (A) are glued together with the passive chip (B) and $\mathrm{Al}$ wire bonding is realized. After the mechanical milling of the edges the device is coated with Parylene C, electrodes are functionalized with MWCNTs and the device is covered with three layers of a biocompatible membrane $(\mathrm{C})$ as described in the next section.

\section{B. Biocompatible Packaging}

Potential sources of inflammation for implantable devices are attributed to the materials, shape and sizes of the devices [5]. MWCNTs were entrapped in a chitosan matrix to prevent toxicity due to the nano-particle nature of the MWCNTs and residues of the catalysts as well. To prevent leaking of potential hazardous substances and the corrosion of electronic components in contact with biologic fluids, a 10- $\mu \mathrm{m}$ coating of Parylene C was employed [10]. For an implantable sensor, the development of a biocompatible packaging is essential for promoting wound healing, and ensuring prolonged sensor functionality. Most of biocompatible packaging from literature [9] require a mold (e.g. for packaging in PDMS) or the application of an additional membrane (e.g. polycarbonate

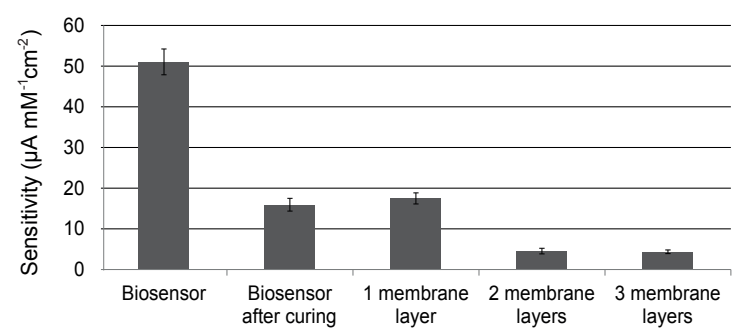

Fig. 3. Heat and membrane thickness effects on the sensor sensitivity (Error bars: standard error of three different SPE-based biosensors). membrane) that results in many manipulation steps that could affect the final biocompatibility grade. In this work, we developed a biocompatible packaging using an epoxy enhanced polyurethane membrane tested for the first time by $\mathrm{Yu}$ et al. [3]. Moreover the porosity of the membrane controls the passage of analytes to the electrodes, without the need of an additional membrane.

1) Membrane characterization and measurements: In-vitro characterization of the membrane was performed on carbonpaste SPEs, in order to assess the optimized number of membrane layers and to understand the membrane effect on enzyme activity. After the functionalization with MWCNTs and glucose oxidase, electrodes were covered with 1-2-3 layers of the membrane, and finally tested for glucose sensitivity with chronoamperometry at $+650 \mathrm{mV}$. Fig. 3 reports the effect of the curing temperature and membrane thickness on sensor sensitivity. The high temperature effect on biosensors without any membrane was investigated, showing a decrease in sensitivity probably due to a partial enzyme denaturation. However LOD was lower after the curing $(18 \pm 1 \mu \mathrm{M})$ compared to the case of a SPE-MWCNT/GOD biosensor $(73 \pm 8$ $\mu \mathrm{M}$ ), due to a reduction of the standard deviation of the background signal (data not shown). Last columns in Fig. 3 show that the application of one layer of membrane does not significantly change sensor performances, probably due to a not homogeneous coating. Two and three layers decrease the sensor sensitivity and increase the LOD $(90 \pm 23 \mu \mathrm{M}$ and $72.0 \pm 0.7 \mu \mathrm{M}$ respectively), but are the best trade-off between the decrease of sensitivity and a homogeneous cover. We also performed chronoamperometry with our microfabricated passive chip. Working electrodes of $1.2 \mathrm{~mm}$ diameter, modified with MWCNTs in chitosan and glucose oxidase, were covered with 2-3 layers of the membrane. All the chips were tested

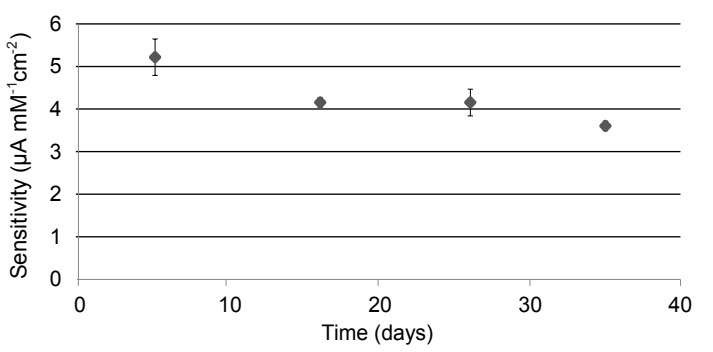

Fig. 4. Long term stability of a biosensor coated by 2 membrane layers (Error bars: standard error of two measurements on each day). 


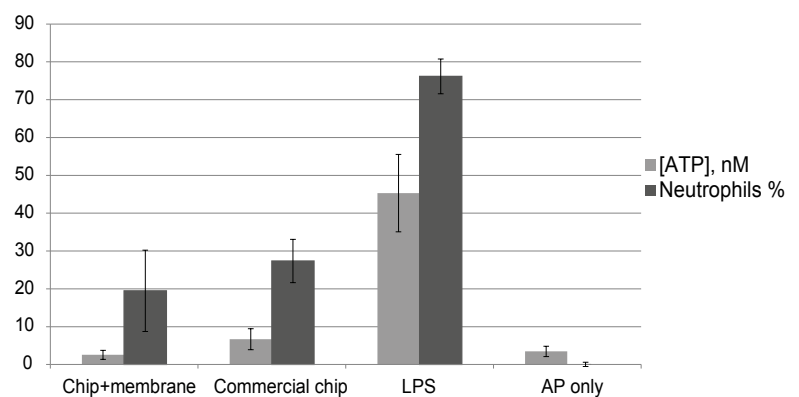

Fig. 5. ATP concentrations (nM) and percentages of neutrophils recovered from APs treated as indicated.

for glucose sensitivity with chronoamperometry at $+650 \mathrm{mV}$, and the sensitivity was then compared. Without any membrane we obtained a sensitivity of $63 \pm 15 \mu \mathrm{A} / \mathrm{mM} \cdot \mathrm{cm}^{2}$ and a LOD of $8 \pm 2 \mu \mathrm{M}$. With 2 layers of the membrane we obtained a lower sensitivity $\left(28 \pm 4 \mu \mathrm{A} / \mathrm{mM} \cdot \mathrm{cm}^{2}\right)$ but a lower LOD $(6 \pm 4 \mu \mathrm{M})$ due again to reduction of the standard deviation of the background signal. Similarly to SPE based sensor, the addition of a third membrane layer does not significantly affect the sensor performances: with 3 layers we obtained a sensitivity of $29 \pm 1 \mu \mathrm{A} / \mathrm{mM} \cdot \mathrm{cm}^{2}$ and a LOD of $7 \pm 4 \mu \mathrm{M}$. The long term stability of the coated biosensor was evaluated by chronoamperometry for glucose sensitivity on SPEs for 40 days. The work in [17] reported a long term stability of 24 days using the same SPE and glucose oxidase immobilized by adsorption onto MWCNT-working electrode. Fig. 4 shows that with two layers of epoxy enhanced polyurethane membrane the sensitivity slightly decreases with respect to time; the relative variation is equal to $28 \%$ after 35 days.

2) Biocompatibility in-vivo tests: In order to investigate the in-vivo biocompatibility of the implantable sensor, we subcutaneously implanted four prototypes in mice for 30 days. At the end of the period, the implant site was washed with PBS, and levels of ATP and neutrophils in the elution liquid were quantified to follow the local inflammatory response. Changes in ATP concentration and changes in neutrophils percentage give information on the local cell death and on the status of tissue inflammation, respectively. Fig. 5 reports ATP and neutrophil variations in the liquid collected from the implant site. ATP and neutrophil levels were also evaluated for a commercial chip (DATA MARS), in case of induced inflammation (LPS) and for the negative control (mice with AP). For the negative control, the neutrophil level is zero while there is still a small amount of extracellular ATP due to the small wound created by the air pouch. Data from both neutrophil infiltration and ATP concentration suggest that the membrane provides a quite good biocompatible coverage. After 30 days, ATP and neutrophil levels are comparable with the negative control (AP), as well as for the commercial chip, and significantly lower than the positive control (LPS), proving that after 30 days the host seems to accept the implant. Unfortunately, a cell layer covered the surface of the sensing platform. Future steps will be the evaluation of the effect of this cell layer on sensing performances and the application of an anti-fouling agent on top of the membrane in order to reduce the cell adhesion.

\section{COnClusions}

In this work we showed the assembly into a fullyimplantable device of a microfabricated sensing platform, a coil for power and data transmission and integrated circuits. We described a device intended to test the biocompatibility of the material used for the microfabrication. Therefore, no electronics measurements are reported here, while data communication and remote powering measurements are the objective of another paper [1]. Experimental results on biosensing with the integrated device are ongoing. An epoxy enhanced polyurethane membrane was used to cover the device to ensure biocompatibility. An in-vitro characterization proved that the membrane is capable to retain enzyme activity up to 35 days. In-vivo experiment in mice proved that, after 30 days of implant, the membrane promotes the integration of the sensor with the surrounding tissue, as demonstrated by the low inflammation levels at the implant site. In conclusions the epoxy enhanced polyurethane membrane is a promising material for the realization of a biocompatible packaging for implantable biosensors.

\section{ACKNOWLEDGMENTS}

The SNF Sinergia Project (CRSII2_127547/1) and NanoSys project (ERC-2009-AdG-246810) financially supported this research.

\section{REFERENCES}

[1] S. Ghoreishizadeh, E. G. Kilinc, C. Baj-Rossi, G. De Micheli, C. Dehollain, and S. Carrara, in IEEE Biomedical Circuits and Systems Conference (BioCAS 2013), Accepted.

[2] F. Valgimigli, F. Lucarelli, C. Scuffi, S. Morandi, and I. Sposato, Journal of diabetes science and technology, vol. 4, no. 5, p. 1182, 2010

[3] B. Yu, N. Long, Y. Moussy, and F. Moussy, Biosensors and Bioelectronics, vol. 21, no. 12, pp. 2275-2282, 2006.

[4] D. A. Gough, L. S. Kumosa, T. L. Routh, J. T. Lin, and J. Y. Lucisano, Science Translational Medicine, vol. 2, no. 42, 2010.

[5] S. Arens, U. Schlegel, G. Printzen, W. Ziegler, S. Perren, and M. Hansis, Journal of Bone \& Joint Surgery, vol. 78, pp. 647-651, 1996.

[6] A. Guiseppi-Elie, C. Lei, and R. H. Baughman, Nanotechnology, vol. 13, no. 5,2002

[7] V. V. Shumyantseva, S. Carrara, V. Bavastrello, D. Jason Riley, T. V. Bulko, K. G. Skryabin, A. I. Archakov, and C. Nicolini, Biosensors and Bioelectronics, vol. 21, no. 1, pp. 217-222, 2005.

[8] V. Erokhin, S. Carrara, H. Amenitch, S. Bernstorff, and C. Nicolini, Nanotechnology, vol. 9, no. 3, pp. 158-163, 1998.

[9] S. Vaddiraju, I. Tomazos, D. J. Burgess, F. C. Jain, and F. Papadimitrakopoulos, Biosensors and Bioelectronics, vol. 25, no. 7, pp. 15531565,32010

[10] A. Cavallini, C. Baj-Rossi, S. Ghoreishizadeh, G. De Micheli, and S. Carrara, in IEEE Biomedical Circuits and Systems Conference (BioCAS 2012), Hsinchu, Taiwan, 2012, pp. 124-127.

[11] S. Carrara, A. Cavallini, V. Erokhin, and G. De Micheli, Biosensors and Bioelectronics, vol. 26, no. 9, pp. 3914-3919, 52011.

[12] J. Mocak, A. Bond, S. Mitchell, and G. Scollary, Pure and Applied Chemistry, vol. 69, no. 2, pp. 297-328, 1997.

[13] S. Carrara, M. D. Torre, A. Cavallini, D. De Venuto, and G. De Micheli, in IEEE Biomedical Circuits and Systems Conference (BioCAS 2010), Paphos, Cyprus, 2010, pp. 146-149.

[14] S. Ghoreishizadeh, S. Carrara, and G. De Micheli, European conference in circuit theory and design (ECCTD), 2013.

[15] E. G. Kilinc, F. Maloberti, and C. Dehollain, in IEEE Biomedical Circuits and Systems Conference (BioCAS 2012), Hsinchu, Taiwan, 2012, pp. 260-263.

[16] E. G. Kilinc, M. A. Ghanad, F. Maloberti, and C. Dehollain, in Proc. NEWCAS13, accepted for publication, Ed., 2013.

[17] C. Boero, S. Carrara, and G. De Micheli, Sensors \& Transducers, vol. 125, no. 2, 2011. 